\title{
FAST PARSING USING PRUNING AND GRAMMAR SPECIALIZATION
}

\author{
Manny Rayner and David Carter \\ SRI International \\ Suite 23, Millers Yard \\ Cambridge CB2 1RQ \\ United Kingdom \\ manny@cam.sri.com, dmcQcam.sri.com
}

\begin{abstract}
We show how a general grammar may be automatically adapted for fast parsing of utterances from a specific domain by means of constituent pruning and grammar specialization based on explanation-based learning. These methods together give an order of magnitude increase in speed, and the coverage loss entailed by grammar specialization is reduced to approximately half that reported in previous work. Experiments described here suggest that the loss of coverage has been reduced to the point where it no longer causes significant performance degradation in the context of a real application.
\end{abstract}

\section{Introduction}

Suppose that we have a general grammar for English, or some other natural language; by this, we mean a grammar which encodes most of the important constructions in the language, and which is intended to be applicable to a large range of different domains and applications. The basic question attacked in this paper is the following one: can such a grammar be concretely useful if we want to process input from a specific domain? In particular, how can a parser that uses a general grammar achieve a level of efficiency that is practically acceptable?

The central problem is simple to state. By the very nature of its construction, a general grammar allows a great many theoretically valid analyses of almost any non-trivial sentence. However, in the context of a specific domain, most of these will be extremely implausible, and can in practice be ignored. If we want efficient parsing, we want to be able to focus our search on only a small portion of the space of theoretically valid grammatical analyses.
One possible solution is of course to dispense with the idea of using a general grammar, and simply code a new grammar for each domain. Many people do this, but one cannot help feeling that something is being missed; intuitively, there are many domain-independent grammatical constraints, which one would prefer only to need to code once. In the last ten years, there have been a number of attempts to find ways to automatically adapt a general grammar and/or parser to the sub-language defined by a suitable training corpus. For example, (Briscoe and Carroll, 1993) train an LR parser based on a general grammar to be able to distinguish between likely and unlikely sequences of parsing actions; (Andry et al., 1994) automatically infer sortal constraints, that can be used to rule out otherwise grammatical constituents; and (Grishman et al., 1984) describes methods that reduce the size of a general grammar to include only rules actually useful for parsing the training corpus.

The work reported here is a logical continuation of two specific strands of research aimed in this general direction. The first is the popular idea of statistical tagging e.g. (DeRose, 1988; Cutting et al, 1992; Church, 1988). Here, the basic idea is that a given small segment $S$ of the input string may have several possible analyses; in particular, if $S$ is a single word, it may potentially be any one of several parts of speech. However, if a substantial training corpus is available to provide reasonable estimates of the relevant parameters, the immediate context surrounding $S$ will usually make most of the locally possible analyses of $S$ extremely implausible. In the specific case of part-of-speech tagging, it is well-known (DeMarcken, 1990) that a large proportion of the incorrect tags can be eliminated "safely", i.e. with very low risk of eliminating correct tags. In the present paper, the statistical tagging idea is generalized to a method called "constituent pruning"; this acts on local analyses of phrases normally 
larger than single-word units.

Constituent pruning is a bottom-up approach, and is complemented by a second, top-down, method based on Explanation-Based Learning (EBL; (Mitchell et al., 1986; van Harmelen and Bundy, 1988)). This part of the paper is essentially an extension and generalization of the line of work described in (Rayner, 1988; Rayner and Samuelsson, 1990; Samuelsson and Rayner, 1991; Rayner and Samuelsson, 1994; Samuelsson, 1994b). Here, the basic idea is that grammar rules tend in any specific domain to combine much more frequently in some ways than in others. Given a sufficiently large corpus parsed by the original, general, grammar, it is possible to identify the common combinations of grammar rules and "chunk" them into "macro-rules". The result is a "specialized" grammar; this has a larger number of rules, but a simpler structure, allowing it in practice to be parsed very much more quickly using an LRbased method (Samuelsson, 1994a). The coverage of the specialized grammar is a strict subset of that of the original grammar; thus any analysis produced by the specialized grammar is guaranteed to be valid in the original one as well. The practical utility of the specialized grammar is largely determined by the loss of coverage incurred by the specialization process.

The two methods, constituent pruning and grammar specialization, are combined as follows. The rules in the original, general, grammar are divided into two sets, called phrasal and non-phrasal respectively. Phrasal rules, the majority of which define non-recursive noun phrase constructions, are used as they are; non-phrasal rules are combined using EBL into chunks, forming a specialized grammar which is then compiled further into a set of LRtables. Parsing proceeds by interleaving constituent creation and deletion. First, the lexicon and morphology rules are used to hypothesize word analyses. Constituent pruning then removes all sufficiently unlikely edges. Next, the phrasal rules are applied bottom-up, to find all possible phrasal edges, after which unlikely edges are again pruned. Finally, the specialized grammar is used to search for full parses. The scheme is fully implemented within a version of the Spoken Language Translator system (Rayner et al., 1993; Agnäs et al., 1994), and is normally applied to input in the form of small lattices of hypotheses produced by a speech recognizer.

The rest of the paper is structured as follows. Section 2 describes the constituent pruning method. Section 3 describes the grammar specialization method, focusing on how the current work extends and improves on previous results. Section 4 describes experiments where the constituent pruning/grammar specialization method was used on sets of previously unseen speech data. Section 5 concludes and sketches further directions for research, which we are presently in the process of investigating.

\section{Constituent Pruning}

Before both the phrasal and full parsing stages, the constituent table (henceforth, the chart) is pruned to remove edges that are relatively unlikely to contribute to correct analyses.

For example, after the string "Show flight D L three one two" is lexically analysed, edges for " $D$ " and " $\mathrm{L}$ " as individual characters are pruned because another edge, derived from a lexical entry for " $D$ L" as an airline code, is deemed far more plausible. Similarly, edges for "one" as a determiner and as a noun are pruned because, when flanked by two other numbers, "one" is far more likely to function as a number.

Phrasal parsing then creates a number of new edges, including one for "flight D L three one two" as a noun phrase. This edge is deemed far more likely to serve as the basis for a correct full parse than any of the edges spanning substrings of this phrase; those edges, too, are therefore pruned. As a result, full parsing is very quick, and only one analysis (the correct one) is produced for the sentence. In the absence of pruning, processing takes over eight times as long and produces 37 analyses in total.

\subsection{The pruning algorithm}

Our algorithm estimates the probability of correctness of each edge: that is, the probability that the edge will contribute to the correct full analysis of the sentence (assuming there is one), given certain lexical and/or syntactic information about it. Values on each criterion (selection of pieces of information) are derived from training corpora by maximum likelihood estimation followed by smoothing. That is, our estimate for the probability that an edge with property $P$ is correct is (modulo smoothing) simply the number of times edges with property $P$ occur in correct analyses in training divided by the number of times such edges are created during the analysis process in training.

The current criteria are:

- The left bigram score: the probability of correctness of an edge considering only the following data about it:

- its $\operatorname{tag}$ (corresponding to its major category symbol plus, for a few categories, some ad- 
ditional distinctions derived from feature values);

- for a lexical edge, its word or semantic word class (words with similar distributions, such as city names, are grouped into classes to overcome data sparseness); or for a phrasal edge, the name of the final (topmost) grammar rule that was used to create it;

- the tag of a neighbouring edge immediately to its left. If there are several left neighbours, the one giving the highest probability is used.

- The right bigram score: as above, but considering right neighbours.

- The unigram score: the probability of correctness of an edge considering only the tree of grammar rules, with words or word classes at the leaves, that gave rise to it. For a lexical edge, this reduces to its word or word class, and its tag.

Other criteria, such as trigrams and finer-grained tags, are obviously worth investigating, and could be applied straightforwardly within the framework described here.

The minimum score derived from any of the criteria applied is deemed initially to be the score of the constituent. That is, an assumption of full statistical dependence (Yarowsky, 1994), rather than the more common full independence, is made. ${ }^{1}$ When

\footnotetext{
${ }^{1}$ If events $E_{1}, E_{2}, \ldots, E_{n}$ are fully independent, then the joint probability $P\left(E_{1} \wedge \ldots \wedge E_{n}\right)$ is the product of $P\left(E_{1}\right) \ldots P\left(E_{n}\right)$, but if they are maximally dependent, it is the minimum of these values. Of course, neither assumption is any more than an approximation to the truth; but assuming dependence has the advantage that the estimate of the joint probability depends much less strongly on $n$, and so estimates for alternative joint events can be directly compared, without any possibly tricky normalization, even if they are composed of different numbers of atomic events. This property is desirable: different (sub-)paths through a chart may span different numbers of edges, and one can imagine evaluation criteria which are only defined for some kinds of edge, or which often duplicate information supplied by other criteria. Taking minima means that the pruning of an edge results from it scoring poorly on one criterion, regardless of other, possibly good scores assigned to it by other criteria. This fits in with the fact that on the basis of local information alone it is not usually possibly to predict with confidence that a particular edge is highly likely to contribute to the correct analysis (since global factors will also be important) but it often is possible to spot highly unlikely edges. In other words, our training procedure yields far more probability estimates close to zero than close to one.
}

recognizer output is being processed, however, the estimate from each criterion is in fact multiplied by a further estimate derived from the acoustic score of the edge: that is, the score assigned by the speech recognizer to the best-scoring sentence hypothesis containing the word or word string for the edge in question. Multiplication is used here because acoustic and lexicosyntactic likelihoods for a word or constituent would appear to be more nearly fully independent than fully dependent, being based on very different kinds of information.

Next, account is taken of the connectivity of the chart. Each vertex of the chart is labelled with the score of the best path through the chart that visits that vertex. In accordance with the dependence assumption, the score of a path is defined as the minimum of the scores of its component edges. Then the score of each edge is recalculated to be the minimum of its existing score and the scores of its start and end vertices, on the grounds that a constituent, however intrinsically plausible, is not worth preserving if it does not occur on any plausible paths.

Finally, a pruning threshold is calculated as the score of the best path through the chart multiplied by a certain fraction. For the first pruning phase we use $1 / 20$, and for the second, $1 / 150$, although performance is not very sensitive to this. Any constituents scoring less than the threshold are pruned out.

\subsection{Relation to other pruning methods}

As the example above suggests, judicious pruning of the chart at appropriate points can greatly restrict the search space and speed up processing. Our method has points of similarity with some very recent work in Constraint Grammar ${ }^{2}$ and is an alternative to several other, related schemes.

Firstly, a remarked earlier, it generalizes tagging: it not only adjudicates between possible labels for the same word, but can also use the existence of a constituent over one span of the chart as justification for pruning another constituent over another span, normally a subsumed one, as in the " $\mathrm{D} L$ " example. This is especially true in the second stage of pruning, when many constituents of different lengths have been created. Furthermore, it applies equally well to lattices, rather than strings, of words, and can take account of acoustic plausibility as well as syntactic considerations.

Secondly, our method is related to beam search (Woods, 1985). In beam search, incomplete parses of an utterance are pruned or discarded when, on

\footnotetext{
${ }^{2}$ Christer Samuelsson, personal communication, 8th April 1996; see (Karlsson et al., 1995) for background.
} 
some criterion, they are significantly less plausible than other, competing parses. This pruning is fully interleaved with the parsing process. In contrast, our pruning takes place only at certain points: currently before parsing begins, and between the phrasal and full parsing stages. Potentially, as with any generate-and-test algorithm, this can mean efficiency is reduced: some paths will be explored that could in principle be pruned earlier. However, as the results in section 4 below will show, this is not in practice a serious problem, because the second pruning phase greatly reduces the search space in preparation for the potentially inefficient full parsing phase. Our method has the advantage, compared to beam search, that there is no need for any particular search order to be followed; when pruning takes place, all constituents that could have been found at the stage in question are guaranteed already to exist.

Thirdly, our method is a generalization of the strategy employed by (McCord, 1993). McCord interleaved parsing with pruning in the same way as us, but only compared constituents over the same span and with the same major category. Our comparisons are more global and therefore can result in more effective pruning.

\section{Grammar specialization}

As described in Section 1 above, the non-phrasal grammar rules are subjected to two phases of processing. In the first, "EBL learning" phase, a parsed training corpus is used to identify "chunks" of rules, which are combined by the EBL algorithm into single macro-rules. In the second phase, the resulting set of "chunked" rules is converted into LR table form, using the method of (Samuelsson, 1994a).

There are two main parameters that can be adjusted in the EBL learning phase. Most simply, there is the size of the training corpus; a larger training corpus means a smaller loss of coverage due to grammar specialization. (Recall that grammar specialization in general trades coverage for speed). Secondly, there is the question of how to select the rulechunks that will be turned into macro-rules. At one limit, the whole parse-tree for each training example is turned into a single rule, resulting in a specialized grammar all of whose derivations are completely "flat". These grammars can be parsed extremely quickly, but the coverage loss is in practice unacceptably high, even with very large training corpora. At the opposite extreme, each rule-chunk consists of a single rule-application; this yields a specialized grammar identical to the original one. The challenge is to find an intermediate solution, which specializes the grammar non-trivially without losing too much coverage.

Several attempts to find good "chunking criteria" are described in the papers by Rayner and Samuelsson quoted above. In (Rayner and Samuelsson, 1994), a simple scheme is given, which creates rules corresponding to four possible units: full utterances, recursive NPs, PPs, and non-recursive NPs. A more elaborate scheme is given in (Samuelsson, 1994b), where the "chunking criteria" are learned automatically by an entropy-minimization method; the results, however, do not appear to improve on the earlier ones. In both cases, the coverage loss due to grammar specialization was about 10 to $12 \%$ using training corpora with about 5,000 examples. In practice, this is still unacceptably high for most applications.

Our current scheme is an extension of the one from (Rayner and Samuelsson, 1994), where the rulechunks are trees of non-phrasal rules whose roots and leaves are categories of the following possible types: full utterances, utterance units, imperative VPs, NPs, relative clauses, VP modifiers and PPs. The resulting specialized grammars are forced to be non-recursive, with derivations being a maximum of six levels deep. This is enforced by imposing the following dominance hierarchy between the possible categories:

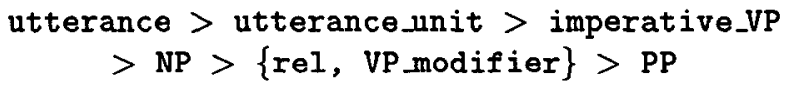

The precise definition of the rule-chunking criteria is quite simple, and is reproduced in the appendix.

Note that only the non-phrasal rules are used as input to the chunks from which the specialized grammar rules are constructed. This has two important advantages. Firstly, since all the phrasal rules are excluded from the specialization process, the coverage loss associated with missing combinations of phrasal rules is eliminated. As the experiments in the next section show, the resulting improvement is quite substantial. Secondly, and possibly even more importantly, the number of specialized rules produced by a given training corpus is approximately halved. The most immediate consequence is that much larger training corpora can be used before the specialized grammars produced become too large to be handled by the LR table compiler. If both phrasal and non-phrasal rules are used, we have been unable to compile tables for rules derived from training sets of over 6,000 examples (the process was killed after running for about six hours on a Sun Sparc 20/HS21, SpecINT92 $=131.2$ ). Using only non-phrasal rules, compilation of the tables for a 15,000 example train- 
ing set required less than two CPU-hours on the same machine.

\section{Experiments}

This section describes a number of experiments carried out to test the utility of the theoretical ideas presented above. The basic corpus used was a set of 16,000 utterances from the Air Travel Planning (ATIS; (Hemphill et al., 1990)) domain. All of these utterances were available in text form; 15,000 of them were used for training, with 1,000 held out for test purposes. Care was taken to ensure not just that the utterances themselves, but also the speakers of the utterances were disjoint between test and training data; as pointed out in (Rayner et al., 1994a), failure to observe these precautions can result in substantial spurious improvements in test data results.

The 16,000 sentence corpus was analysed by the SRI Core Language Engine (Alshawi (ed), 1992), using a lexicon extended to cover the ATIS domain (Rayner, 1994). All possible grammatical analyses of each utterance were recorded, and an interactive tool was used to allow a human judge to identify the correct and incorrect readings of each utterance. The judge was a first-year undergraduate student with a good knowledge of linguistics but no prior experience with the system; the process of judging the corpus took about two and a half person-months. The input to the EBL-based grammar-specialization process was limited to readings of corpus utterances that had been judged correct. When utterances had more than one correct reading, a preference heuristic was used to select the most plausible one.

Two sets of experiments were performed. In the first, increasingly large portions of the training set were used to train specialized grammars. The coverage loss due to grammar specialization was then measured on the 1,000 utterance test set. The experiment was carried out using both the chunking criteria from (Rayner and Samuelsson, 1994) (the "Old" scheme), and the chunking criteria described in Section 3 above (the "New" scheme). The results are presented in Table 1.

The second set of experiments tested more directly the effect of constituent pruning and grammar specialization on the Spoken Language Translator's speed and coverage; in particular, coverage was measured on the real task of translating English into Swedish, rather than the artificial one of producing a correct QLF analysis. To this end, the first 500 testset utterances were presented in the form of speech hypothesis lattices derived by aligning and conflating the top five sentence strings produced by a version of the DECIPHER (TM) recognizer (Murveit

\begin{tabular}{|r|r|r|r|r|}
\hline Examples & \multicolumn{2}{|c|}{ Old scheme } & \multicolumn{2}{c|}{ New scheme } \\
\hline & Rules & Loss & Rules & Loss \\
\hline 100 & 100 & $47.8 \%$ & 69 & $35.5 \%$ \\
250 & 181 & $37.6 \%$ & 126 & $21.8 \%$ \\
500 & 281 & $27.6 \%$ & 180 & $14.7 \%$ \\
1000 & 432 & $22.7 \%$ & 249 & $10.8 \%$ \\
3000 & 839 & $14.9 \%$ & 455 & $7.8 \%$ \\
5000 & 1101 & $11.2 \%$ & 585 & $6.6 \%$ \\
7000 & 1292 & $10.4 \%$ & 668 & $6.0 \%$ \\
11000 & 1550 & $9.8 \%$ & 808 & $5.8 \%$ \\
15000 & 1819 & $8.7 \%$ & 937 & $5.0 \%$ \\
\hline
\end{tabular}

Table 1: EBL rules and EBL coverage loss against number of training examples

et al., 1993). The lattices were analysed by four different versions of the parser, exploring the different combinations of turning constituent pruning on or off, and specialized versus unspecialized grammars. The specialized grammar used the "New" scheme, and had been trained on the full training set. Utterances which took more than $90 \mathrm{CPU}$ seconds to process were timed out and counted as failures.

The four sets of outputs from the parser were then translated into Swedish by the SLT transfer and generation mechanism (Agnäs et al., 1994). Finally, the four sets of candidate translations were pairwise compared in the cases where differing translations had been produced. We have found this to be an effective way of evaluating system performance. Although people differ widely in their judgements of whether a given translation can be regarded as "acceptable", it is in most cases surprisingly easy to say which of two possible translations is preferable. The last two tables summarize the results. Table 2 gives the average processing times per input lattice for each type of processing (times measured running SICStus Prolog $3 \# 3$ on a SUN Sparc 20/HS21), showing how the time is divided between the various processing phases. Table 3 shows the relative scores of the four parsing variants, measured according to the "preferable translation" criterion.

\section{Conclusions and further directions}

Table 2 indicates that EBL and pruning each make processing about three times faster; the combination of both gives a factor of about nine. In fact, as the detailed breakdown shows, even this underestimates the effect on the main parsing phase: when both pruning and EBL are operating, processing times for other components (morphology, pruning and preferences) become the dominant ones. As we have so 


\begin{tabular}{|r|r|r|r|r|}
\hline & $\begin{array}{r}\text { E- } \\
\text { P- }\end{array}$ & $\begin{array}{r}\text { E+ } \\
\text { P- }\end{array}$ & $\begin{array}{c}\text { E- } \\
\text { P+ }\end{array}$ & $\begin{array}{c}\text { E+ } \\
\text { P+ }\end{array}$ \\
\hline Morph/lex lookup & 0.53 & 0.54 & 0.54 & 0.49 \\
\hline Phrasal parsing & 0.27 & 0.28 & 0.14 & 0.14 \\
\hline Pruning & - & - & 0.57 & 0.56 \\
\hline Full parsing & 12.42 & 2.61 & 3.04 & 0.26 \\
\hline Preferences & 3.63 & 1.57 & 1.27 & 0.41 \\
\hline \hline TOTAL & 16.85 & 5.00 & 5.57 & 1.86 \\
\hline
\end{tabular}

Table 2: Breakdown of average time spent on each processing phase for each type of processing (seconds per utterance)

\begin{tabular}{|c|c|c|c|c|}
\hline & $\begin{array}{c}\text { E- } \\
\text { P- }\end{array}$ & $\begin{array}{c}\text { E+ } \\
\text { P- }\end{array}$ & $\begin{array}{c}\text { E- } \\
\text { P+ }\end{array}$ & $\begin{array}{c}\text { E+ } \\
\text { P+ }\end{array}$ \\
\hline $\mathrm{E}-/ \mathrm{P}-$ & & $12-24$ & $25-63$ & $24-65$ \\
\hline $\mathrm{E}+/ \mathrm{P}-$ & $24-12$ & & $31-50$ & $26-47$ \\
\hline $\mathrm{E}-/ \mathrm{P}+$ & $63-25$ & $50-31$ & & $5-8$ \\
\hline $\mathrm{E}+/ \mathrm{P}+$ & $65-24$ & $47-26$ & $8-5$ & \\
\hline
\end{tabular}

Table 3: Comparison between translation results on the four different analysis alternatives, measured on the 500-utterance test set. The entry for a given row and column holds two figures, showing respectively the number of examples where the "row" variant produced a better translation than the "column" variant and the number where it produced a worse one. Thus for example "EBL+/pruningt" was better than "EBL-/pruning-" on 65 examples, and worse on 24 .

far expended little effort on optimizing these phases of processing, it is reasonable to expect substantial further gains to be possible.

Even more interestingly, Table 3 shows that real system performance, in terms of producing a good translation, is significantly improved by pruning, and is not degraded by grammar specialization. (The slight improvement in coverage with EBL on is not statistically significant). Our interpretation of these results is that the technical loss of grammar coverage due to the specialization and pruning processes is more than counterbalanced by two positive effects. Firstly, fewer utterances time out due to slow processing; secondly, the reduced space of possible analyses means that the problem of selecting between different possible analyses of a given utterance becomes easier.

To sum up, the methods presented here demonstrate that it is possible to use the combined pruning and grammar specialization method to speed up the whole analysis phase by nearly an order of magni- tude, without incurring any real penalty in the form of reduced coverage. We find this an exciting and significant result, and are further continuing our research in this area during the coming year. In the last two paragraphs we sketch some ongoing work.

All the results presented above pertain to English only. The first topic we have been investigating is the application of the methods described here to processing of other languages. Preliminary experiments we have carried out on the Swedish version of the CLE (Gambäck and Rayner 1992) have been encouraging; using exactly the same pruning methods and EBL chunking criteria as for English, we obtain comparable speed-ups. The loss of coverage due to grammar specialization also appears comparable, though we have not yet had time to do the work needed to verify this properly. We intend to do so soon, and also to repeat the experiments on the French version of the CLE (Rayner, Carter and Bouillon, 1996).

The second topic is a more radical departure, and can be viewed as an attempt to make interleaving of parsing and pruning the basic principle underlying the CLE's linguistic analysis process. Exploiting the "stratified" nature of the EBL-specialized grammar, we group the chunked rules by level, and apply them one level at a time, starting at the bottom. After each level, constituent pruning is used to eliminate unlikely constituents. The intent is to achieve a trainable robust parsing model, which can return a useful partial analysis when no single global analysis is found. An initial implementation exists, and is currently being tested; preliminary results here are also very positive. We expect to be able to report on this work more fully in the near future.

\section{Acknowledgements}

The work reported in this paper was funded by Telia Research AB. We would like to thank Christer Samuelsson for making the LR compiler available to us, Martin Keegan for patiently judging the results of processing 16,000 ATIS utterances, and Steve Pulman and Christer Samuelsson for helpful comments.

\section{References}

Agnäs, M-S., Alshawi, H., Bretan, I., Carter, D.M. Ceder, K., Collins, M., Crouch, R., Digalakis, V., Ekholm, B., Gambäck, B., Kaja, J., Karlgren, J., Lyberg, B., Price, P., Pulman, S., Rayner, M., Samuelsson, C. and Svensson, T. 1994. Spoken 
Language Translator: First Year Report. SRI technical report CRC- $043^{3}$

Alshawi, H. (ed.) 1992. The Core Language Engine. MIT Press.

Andry, F., M. Gawron, J. Dowding, and R. Moore. 1994. A Tool for Collecting Domain Dependent Sortal Constraints From Corpora. Proc. COLING-94, Kyoto.

Briscoe, Ted, and John Carroll. 1993. Generalized Probabilistic LR Parsing of Natural Language (Corpora) with Unification-Based Grammars. Computational Linguistics, 19:1, pp. 25-60.

Church, Ken. 1988. A stochastic parts program and noun phrase parser for unrestricted text. Proc. 1st ANLP, Austin, Tx., pp. 136-143.

Cutting, D., J. Kupiec, J. Pedersen and P. Sibun. 1992. A Practical Part-of-Speech Tagger Proc. 3rd ANLP, Trento, Italy, pp. 133-140.

DeMarcken, C.G. 1990. Parsing the LOB Corpus Proc. 28th ACL, Pittsburgh, Pa., pp. 243-251

DeRose, Steven. 1988. Grammatical Category Disambiguation by Statistical Optimization. Computational Linguistics 14, pp. 31-39

Gambäck, Björn, and Manny Rayner. "The Swedish Core Language Engine". Proc. 3rd Nordic Conference on Text Comprehension in Man and Machine, Linköping, Sweden. Also SRI Technical Report CRC-025.

Grishman, R., N. Nhan, E. Marsh and L. Hirschmann. 1984. Automated Determination of Sublanguage Usage. Proc. 22nd COLING, Stanford, pp. 96-100.

van Harmelen, Frank, and Alan Bundy. 1988. Explanation-Based Generalization = Partial Evaluation (Research Note) Artificial Intelligence 36, pp. 401-412.

Hemphill, C.T., J.J. Godfrey and G.R. Doddington. 1990. The ATIS Spoken Language Systems pilot corpus. Proc. DARPA Speech and Natural Language Workshop, Hidden Valley, Pa., pp. 96-101.

Karlsson, F., A. Voutilainen, J. Heikkilä and A. Anttila (eds). 1995. Constraint Grammar. Mouton de Gruyer, Berlin, New York.

McCord, M. 1993. Heuristics for BroadCoverage Natural Language Parsing. Proc. 1st ARPA Workshop on Human Language Technology, Princeton, NJ. Morgan Kaufmann.

\footnotetext{
${ }^{3}$ All SRI Cambridge technical reports are available through WWW from http://ww. cam.sri.com
}

Mitchell, T., R. Keller, and S. Kedar-Cabelli. 1986. Explanation-Based Generalization: a Unifying View. Machine Learning 1:1, pp. 47-80.

Murveit, H., Butzberger, J., Digalakis, V. and Weintraub, M. 1993. Large Vocabulary Dictation using SRI's DECIPHER(TM) Speech Recognition System: Progressive Search Techniques. Proc. Inter. Conf. on Acoust., Speech and Signal, Minneapolis, Mn.

Rayner, M. 1988. Applying Explanation-Based Generalization to Natural-Language Processing. Proc. the International Conference on Fifth Generation Computer Systems, Kyoto, pp. 1267-1274.

Rayner, M. 1994. Overview of English Linguistic Coverage. In (Agnäs et al., 1994)

Rayner, M., Alshawi, H., Bretan, I., Carter, D.M., Digalakis, V., Gambäck, B., Kaja, J., Karlgren, J., Lyberg, B., Price, P., Pulman, S. and Samuelsson, C. 1993. A Speech to Speech Translation System Built From Standard Components. Proc.1st ARPA workshop on Human Language Technology, Princeton, NJ. Morgan Kaufmann. Also SRI Technical Report CRC-031.

Rayner, M., D. Carter and P. Bouillon. 1996. Adapting the Core Language Engine to French and Spanish. Proc. NLP-IA, Moncton, New Brunswick. Also SRI Technical Report CRC-061.

Rayner, M., D. Carter, V. Digalakis and P. Price. 1994. Combining Knowledge Sources to Reorder N-Best Speech Hypothesis Lists. Proc.2̃nd ARPA workshop on Human Language Technology, Princeton, NJ., pp. 217-221. Morgan Kaufmann. Also SRI Technical Report CRC-044.

Rayner. M., and C. Samuelsson. 1990. Using Explanation-Based Learning to Increase Performance in a Large NL Query System. Proc. DARPA Speech and Natural Language Workshop, June 1990, pp. 251-256. Morgan Kaufmann.

Rayner, M., and C. Samuelsson. 1994. CorpusBased Grammar Specialization for Fast Analysis. In (Agnäs et al., 1994)

Samuelsson, C. 1994. Notes on LR Parser Design. Proc. COLING-94, Kyoto, pp. 386-390.

Samuelsson, C. 1994. Grammar Specialization through Entropy Thresholds. Proc. ACL-94, Las Cruces, NM, pp. 188-195.

Samuelsson, C., and M. Rayner. 1991. Quantitative Evaluation of Explanation-Based Learning as an Optimization Tool for a Large-Scale Natural Language System. Proc. 12th IJCAI, Sydney, pp. 609615 . 
Woods, W. 1985. Language Processing for Speech Understanding. Computer Speech Processing, W. Woods and F. Fallside (eds), Prentice-Hall International.

Yarowsky, D. 1994. Decision Lists for Lexical Ambiguity Resolution. Proc. ACL-94, Las Cruces, NM, pp. 88-95.

\section{Appendix: definition of the "New" chunking rules}

This appendix defines the "New" chunking rules referred to in Sections 3 and 4. There are seven types of non-phrasal constituent in the specialised grammar. We start by describing each type of constituent through examples.

Utterance: The top category.

Utterance_unit: Utterance_ units are minimal syntactic units capable of standing on their own: for example, declarative clauses, questions, NPs and PPs. Utterances may consist of more than one utterance unit. The following is an utterance containing two utteranceunits: "[Flights to Boston on Monday] [please show me the cheapest ones.]"

Imperative_VP: Since imperative verb phrases are very common in the corpus, we make them a category of their own in the specialised grammar. To generalise over possible addition of adverbials (in particular, "please" and "now"), we define the imperative_vp category so as to leave the adverbials outside. Thus the bracketed portion of the following utterance is an imperative_vp: "That's fine now [give me the fares for those flights]"

Non_phrasal_NP: All NPs which are not produced entirely by phrasal rules. The following are all non phrasal NPs: "Boston and Denver", "Flights on Sunday morning", "Cheapest fare from Boston to Denver", "The meal I'd get on that flight"

Rel: Relative clauses.

VP_modifier: VPs appearing as NP postmodifiers. The bracketed portions of the following are VP_modifiers: "Delta flights [arriving after seven P M]" "All flights tomorrow [ordered by arrival time]"

PP: The CLE grammar treats nominal temporal adverbials, sequences of PPs, and "A to B" constructions as PPs (cf (Rayner, 1994)). The following are examples of PPs: "Tomorrow afternoon", "From Boston to Dallas on Friday", "Denver to San Francisco Sunday"

We can now present the precise criteria which determine the chunks of rules composed to form each type of constituent. For each type of constituent in the specialised grammar, the chunk is a subtree extracted from the derivation tree of a training example (cf (Rayner and Samuelsson, 1994)); we specify the roots and leaves of the relevant subtrees. The term "phrasal tree" will be used to mean a derivation tree all of whose rule-applications are phrasal rules.

Utterance: The root of the chunk is the root of the original tree. The leaves are the nodes resulting from cutting at maximal subtrees for utteranceunits, non_phrasal_nps pps, and maximal phrasal subtrees.

Utterance_unit: The root is the root of a maximal subtree for a constituent of type utteranceunit. The leaves are the nodes resulting from cutting at maximal subtrees for imperative_vps, nps, and pps, and maximal phrasal subtrees.

Imperative_VP: The root is the root of a maximal subtree under an application of the $S \rightarrow$ VP rule whose root is not an application of an adverbial modification rule. The leaves are the nodes resulting from cutting at maximal subtrees for non_phrasal_np, and pp, and maximal phrasal subtrees.

Non_phrasal_NP: The root is the root of a maximal non-phrasal subtree for a constituent of type np. The leaves are the nodes resulting from cutting at maximal subtrees for rel, vpmodifier, and pp, and maximal phrasal subtrees.

Rel: The root is the root of a maximal subtree for a constituent of type rel. The leaves are the nodes resulting from cutting at maximal subtrees for pp, and maximal phrasal subtrees.

VP_modifier: The root is the root of a vp subtree immediately dominated by an application of the NP $\rightarrow$ NP VP rule. The leaves are the nodes resulting from cutting at maximal subtrees for PP, and maximal phrasal subtrees.

PP: The root is the root of a maximal non-phrasal subtree for a constituent of type pp. The leaves are the nodes resulting from cutting at maximal phrasal subtrees. 